\title{
PULMONARY ATRESIA WITH INTACT VENTRICULAR SEPTUM
}

\author{
BY
}

\section{KARL EDVARD EGENBERG}

From the Laboratory of Clinical Physiology, University Clinic, Bergen, Norway

Pulmonary atresia with intact ventricular septum is a rare type of congenital lesion. Although it has been recognized pathologically for almost two centuries (Hunter, 1784), the few clinical studies available have appeared during recent years (Greenwold et al., 1956; Keith, Rowe, and Vlad, 1958; Paul and Lev, 1960; and Schrire, Sutin, and Barnard, 1961).

\section{Case Report}

A female child, cyanotic from birth and suffering from repeated upper respiratory infections, needed digitalis at the age of 4 months on account of congestive heart failure. During the following months cyanotic attacks developed, and clubbing of the fingers and toes was noted before the age of 6 months.

On examination in November 1961, at the age of 15 months, severe cyanosis and physical underdevelopment were evident. A grade 3 pansystolic murmur was audible over the whole of the præcordium and also to the right of the lower part of the sternum. No splitting of the second sound could be heard. Radiological examination revealed oligæmic lungs and an enlarged cardiac shadow, believed to be due mainly to right-sided enlargement (Fig. 1). Electrocardiographic records from previous medical examinations were available. They showed $P$ waves that were gigantic in some of the leads and bifid in others (Fig. 3).

In the tracings from the neonatal period (September 1960) no abnormal axis deviation was present, and there was an Rs pattern in the lateral præcordial leads. During the ensuing months they revealed the development of right axis deviation and an rS pattern in V5-V6. The main findings on cardiac catheterization are presented in the Table.

TABLE

CATHETERization Data

\begin{tabular}{c|c|c|c|c|c|c|c}
\hline \multicolumn{5}{c|}{ Pressures (mm. Hg) } & \multicolumn{2}{c}{$\begin{array}{c}\text { Oxygen saturation } \\
\text { (per cent) }\end{array}$} \\
\hline RA & $\begin{array}{c}\text { RA } \\
\text { mean }\end{array}$ & LA & $\begin{array}{c}\text { LA } \\
\text { mean }\end{array}$ & RV & LV & RA & LA \\
\hline $17 / 7$ & 10 & $8 / 3$ & 6 & $50 / 6$ & $60 / 3$ & $50 \cdot 7$ & $56 \cdot 0$ \\
\hline
\end{tabular}

Despite repeated attempts the pulmonary trunk could not be entered. Angiocardiography was carried out from the right ventricle (Fig. 2) and revealed a small ventricle from which no passage of contrast medium to the pulmonary trunk could be seen. Retrograde filling of an enlarged right atrium took place, however, and from there the contrast medium entered the left atrium, the left ventricle, and the aorta. The pulmonary vessels were filled from the aorta.

On the diagnosis of pulmonary atresia with intact ventricular septum, the child was transferred to a surgical department for valvotomy, but died before the operation was attempted.

Necropsy revealed the characteristic fusion of the pulmonary cusps to an imperforate diaphragm. The right ventricular cavity was small, the right atrium was dilated with hypertrophic walls, and the foramen 


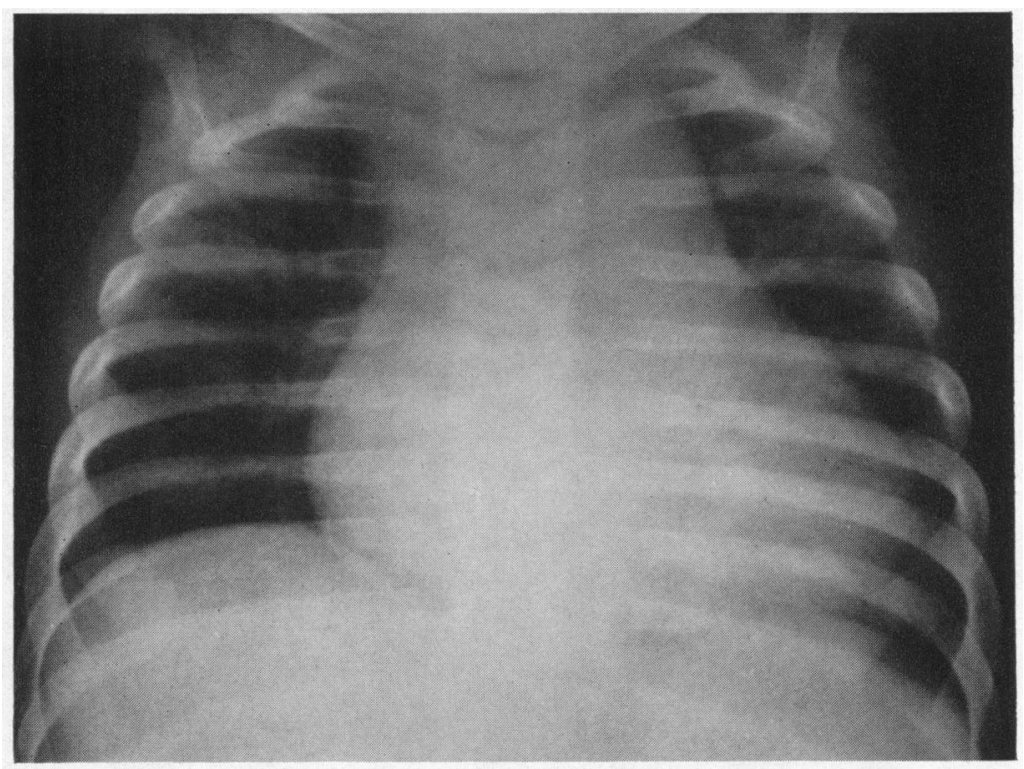

Fig. 1.-Postero-anterior radiograph showing cardiac enlargement.

FIG. 2.-Angiocardiography, left lateral view showing small right ventricle (RV), no passage of contrast to the pulmonary trunk, and retrograde filling of the right atrium (RA). The left atrium (LA) and the left ventricle (LV) are then opacified.

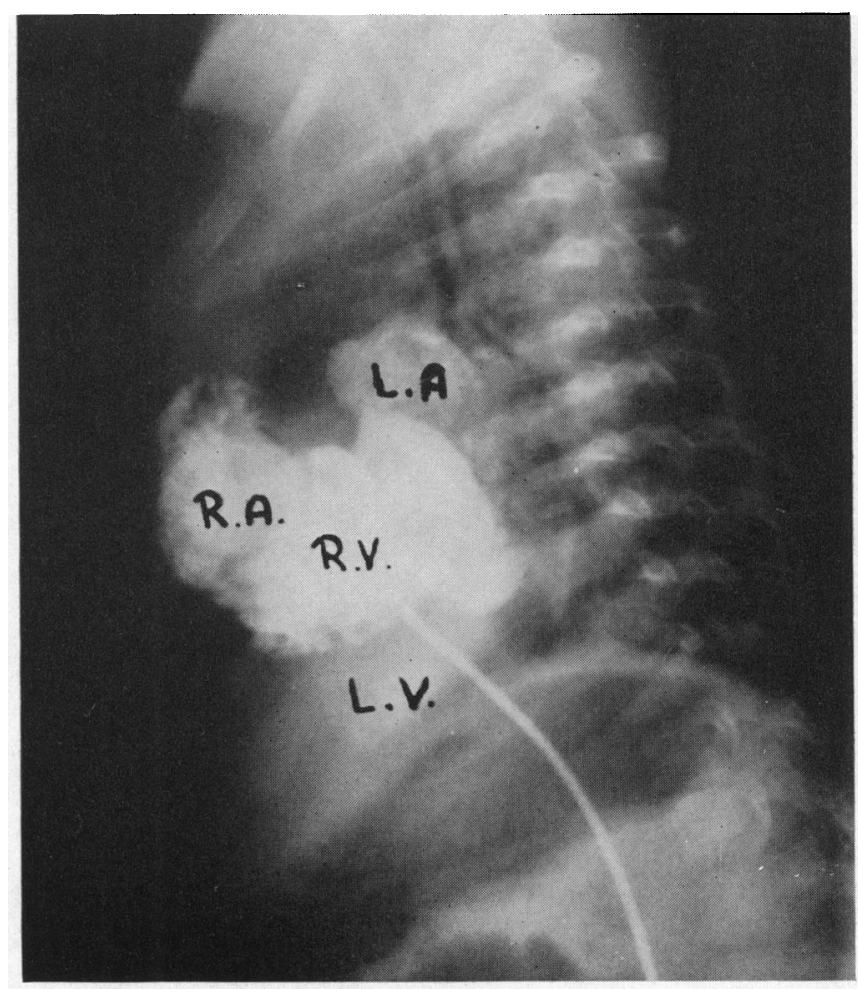




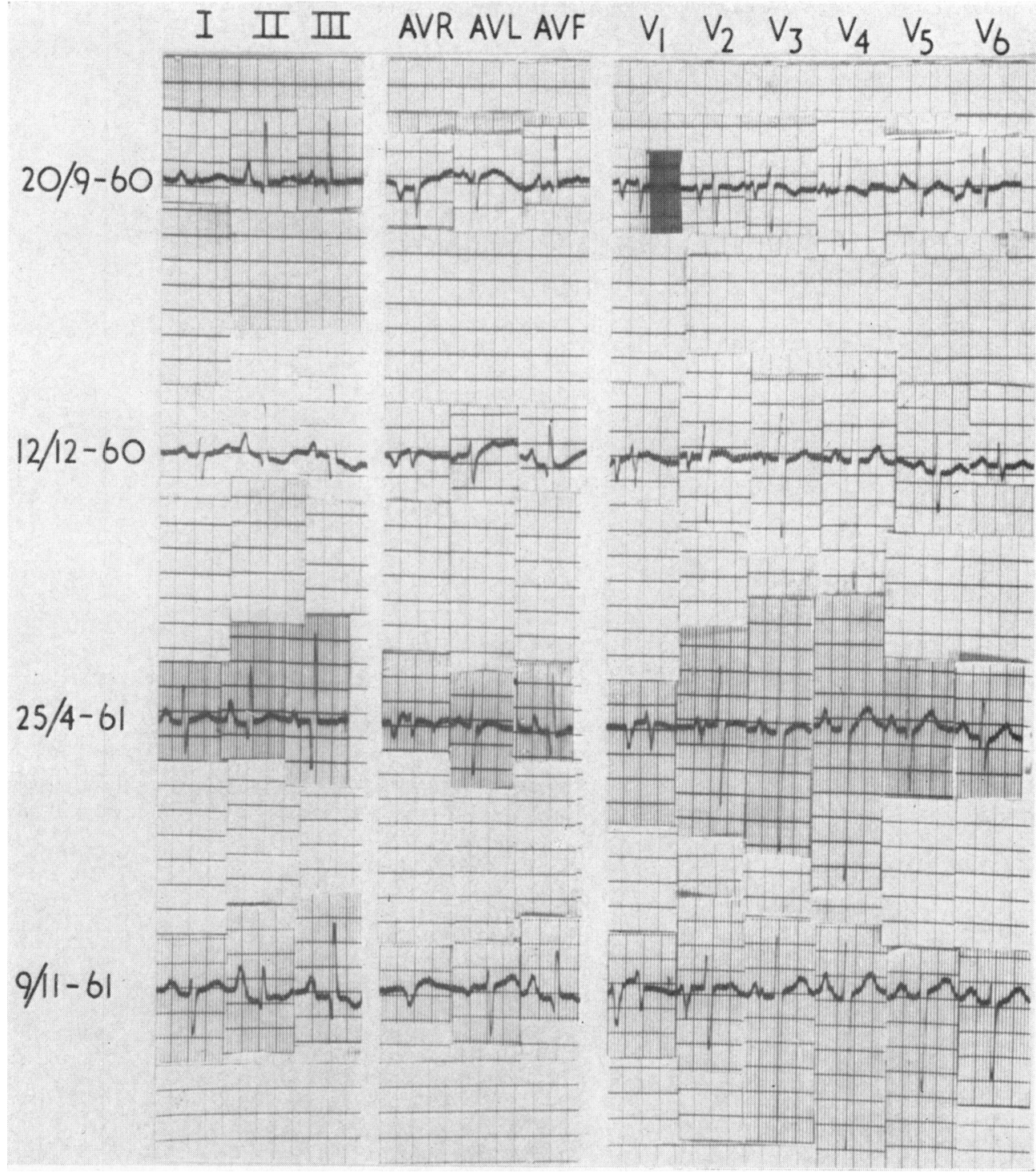

FIG. 3.-Evolution of the electrocardiograms in a case of pulmonary atresia with intact ventricular septum. There is a change in axis from $+85^{\circ}$ to $+120^{\circ}$, development of giant $\mathbf{P}$ waves, and a change from Rs to rS pattern in V5-V6.

ovale was patent. There was no ventricular septal defect. The left ventricle was moderately dilated and hypertrophic. The pulmonary artery was patent but narrow. The aorta was wide, and the ductus arteriosus had remained patent.

\section{Discussion}

Venous blood is shunted from the right to the left side of the heart at atrial level, usually, as in the present patient, through a patent foramen ovale. The lungs receive their blood supply through a patent ductus arteriosus. Greenwold et al. (1956) have stressed that there are two types of this 


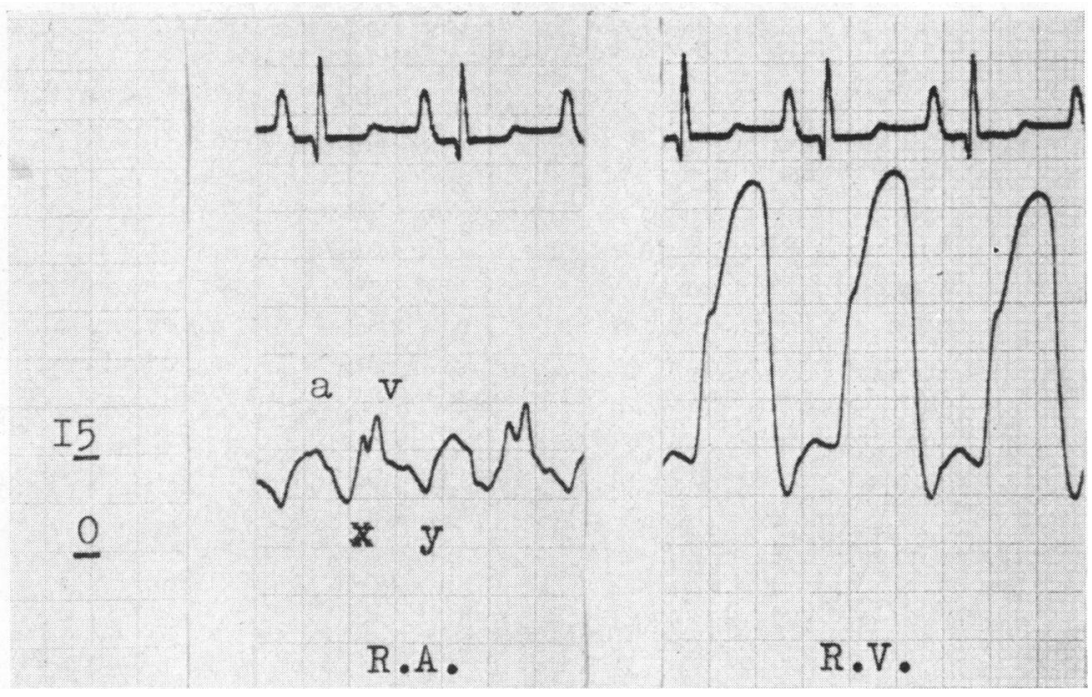

FIG. 4.-Pressure tracings from right atrium (RA) and right ventricle (RV). The large ' $v$ ' wave suggests tricuspid regurgitation.

malformation: in approximately 80 per cent of the cases the right ventricular cavity is small, while in the remainder it is dilated. Muscular hypertrophy is common to both types. The cause of the ventricular dilatation is believed to be tricuspid regurgitation.

The systolic murmur, noted to the right of the lower part of the sternum in our case, and the large "v" wave in the pressure curve from the right atrium (Fig. 4) indicate retrograde movement of blood through the tricuspid valve (Wood, 1956). Thus tricuspid regurgitation may also occur in cases with a small right ventricular cavity. Probably both the duration and the degree of regurgitation are important factors in the development of the right ventricular dilatation, and transition forms between the two main types presumably occur.

Keith et al. (1958) point out that the electrocardiogram is usually sufficiently distinctive to permit of at least a presumptive diagnosis. It may show evidence of right atrial hypertrophy, and a variable degree of left and right ventricular hypertrophy.

An important feature is a change in direction of the axis in the first months of life to the right. This is well shown in the present case by the consecutive electrocardiographic records which show a change in axis direction from $85^{\circ}$ to $120^{\circ}$. The electrical position is vertical, and the tracings show a progressive degree of clockwise rotation. The development of the giant $\mathbf{P}$ waves is also a noteworthy feature. In the last cardiogram the amplitude of the $P$ wave is larger than that of the QRS complex in lead II.

Differentiation between pulmonary atresia with intact ventricular septum and tricuspid atresia may be difficult. In cases of tricuspid atresia, the right ventricle is rudimentary and the cardiographic pattern accordingly shows left axis deviation with horizontal electrical position, left ventricular hypertrophy, and no signs of right ventricular hypertrophy. The appearance of the cardiogram tends to remain unaltered, and no tendency towards development of right axis deviation is encountered. A systolic murmur to the right of the lower part of the sternum, indicating tricuspid regurgitation, may help in excluding the possibility of tricuspid atresia.

Likewise, distinction between pulmonary atresia with closed ventricular septum, and severe pulmonary stenosis with right-to-left shunt at the atrial level, may also prove difficult. The radiological findings may be similar, and in rare cases, especially when the pulmonary stenosis is combined with defective development of the right ventricle, the usual loud and harsh systolic murmur over the 
pulmonary area is absent. Furthermore, in cases with defective development of the right ventricle, the cardiograms mey be misleading by showing only moderate signs of right ventricular hypertrophy, or even left ventricular dominance in the præcordial leads. The two conditions may, admittedly, merge into each other when the pulmonary orifice is practically non-existent. In such cases even angiocardiography may fail to show that the opening is still patent.

The tetralogy of Fallot may be confused with pulmonary atresia with intact ventricular septum. Enlargement of the $\mathrm{P}$ wave is, however, unusual in infants with this malformation, and even in older children the $\mathrm{P}$ waves do not reach the extreme amplitude met with in pulmonary atresia with intact ventricular septum. Transposition of the great vessels may produce a clinical and electrocardiographic picture similar to that of pulmonary atresia with intact ventricular septum. Radiological examination, cardiac catheterization, and angiocardiography will usually demonstrate the abnormal position of the great vessels, however.

Valvotomy should be attempted in cases of pulmonary atresia with intact ventricular septum, the pulmonary trunk being patent in most cases. With surgical treatment the prognosis is still poor, though far from hopeless.

\section{Summary}

A case of pulmonary atresia with intact ventricular septum is described with special reference to the presence of tricuspid regurgitation and to the development of right axis deviation and large right atrial $\mathbf{P}$ waves in sequential cardiograms.

The differential diagnosis from tricuspid atresia, lone pulmonary stenosis, and the tetralogy of Fallot is discussed.

\section{References}

Greenwold, W. E., DuShane, J. W., Burchell, H. B., Bruwer, A., and Edwards, J. E. (1956). Congenital pulmonary atresia with intact ventricular septum: two anatomic types. Circulation, 14, 945.

Hunter, W. (1784). Medical Observations and Inquiries, 6, 291. Cited by Peacock (1869).

Keith, J. D., Rowe, R. D., and Vlad, P. (1958). Heart Disease in Infancy and Childhood, p. 569. Macmillan, New York.

Paul, M. H., and Lev, M. (1960). Tricuspid stenosis with pulmonary atresia. A cineangiographic-pathologic correlation. Circulation, $22,198$.

Schrire, V., Sutin, G. J., and Barnard, C. N. (1961). Organic and functional pulmonary atresia with intact ventricular septum. Amer. J. Cardiol., 8, 100.

Wood, P. (1956). Diseases of the Heart and Circulation, 2nd ed., pp. 584-585. Eyre and Spottiswoode, London. 